\title{
MEGACOLON AND MICROCOLON *
}

\author{
LANGLEY PORTER, M.D., AND ALANSON WEEKS, M.D. \\ SAN FRANCISCO
}

Pediatricians express difference of opinion as to the relative frequency of megacolon. Some authors of wide experience are able to make reports on only one or two such cases; that such experience is not general is attested by the fact that in the literature of the past four years there are more than one hundred papers each of which deals with one to six cases. It follows, therefore, that the affliction cannot be extremely rare. We have encountered six cases during the past five years; two of these passed from observation after one view and, therefore, are not included in this report. Microcolon, on the other hand, seems to be truly an unusual condition, as our search through the literature revealed but three certain and one doubtful case, although it seems probable that some reports under the caption "Stenosis of the Colon" may have referred to this condition.

The name "megacolon" has been used to describe the clinical picture characterized by obstinate constipation, great abdominal enlargement, visible intestinal peristalsis with the constitutional results of toxemia due to fecal retention. Constipation at times causes such retention of feces that fecal masses accumulate; these may shift and cause attacks of acute or subacute obstruction of the bowel. The literature referring to this congenital enlargement of the colon is ample. In his masterly article written in 1908, Finney presents a bibliography of 206 titles compiled by A. W. Fisher, complete to the time of his writing. We have searched the libraries diligently and find that over 100 papers on the subject have appeared since Finney's writing. One of the best of these later articles was written by Barrington-Ward and published in January, 1914. One must agree with Barrington-Ward's statement that Finney's paper so completely discusses all phases of the disease that it must remain the authoritative communication on this subject for a long time to come.

Although Finney shows that some twenty odd cases, one by Lewitt of Chicago (186\%) and another by Jacobi, were clearly described before Hirschsprung published his studiss of the malady in 1896 , we owe the eninent Dane a debt for centcring attention on the importance of the condition. This writer classifierl giant colon as: $(a)$ true megacolon, first noticeable at birth or early infancy; $(b)$ pseudomegacolon, making its first appearance in adult life and showing dilatation of the

* From the Medical Department of Leland Stanford, Jr., University. 
colon with hypertrophy of its muscle, and arising secondary to some partial obstruction that came on during the postnatal period. Many writers deny psetidomegacolon pathologic validity, and, in fact, there seems to be little common to the two conditions.

Barrington-Ward divides the theories which seek to explain the origin of megacolon into four classes: (1) the mechanical; $(2)$ the neuropathic; (3) the inflammatory and (4) the congenital. Under the mechanical theory he arranges five subheads: $(a)$ the view of Barth, that an increased mesentery permits torsions of the sigmoid with partial occlusion and subsequent hypertrophy and gigantism of the intestine; $(b)$ the view of Marfan, that the increased length of the intestine, especially the sigmoid portion, throws the bowel into a loop which causes kinking obstructions; $(c)$ Treves' view, that the obstruction follows atresia of the anus and rectum; $(d)$ Perth's idea, that valve formation is the cause of the obstruction; $(e)$ Wilkie's conception, that the primary obstruction is due to the distention of the pelvic colon with meconium at time of birth or shortly after. As will be seen, none of these theories makes the difficulty of interpretation less.

Four different explanations are offered in elucidation of the neuropathic theory. It is suggested: $(a)$ that there is a neuromuscular defect of the segment of the intestine; $(b)$ that there may be a paralysis of a segment of the intestine; $(c)$ that there is some implication of the sympathetic nervous system, and $(d)$ that reflex spasm of the sphincter ani, a result of a fissure in ano, is the basic cause of the trouble.

Adherents to the third theory hold that a colitis that later becomes chronic originates those pathological processes which afterward produce the full picture of megacolon.

The proponents of the fourth theory assign the only cause which seems to explain the known facts. They hold with Hirschsprung that the condition is an anomaly of development and that the hypertrophy and dilatation both occur in the fetal intestine before birth. Concetti, while acknowledging the congenital origin of the dilatation, considers that the hypertrophy follows effort made to overcome the obstruction due to an inert segment of the dilated bowel. Other writers, among them Fenwick, believe that the hypertrophy is the congenital feature, and that the dilatation is the result of the pressure of the retained gases. In favor of the theory of congenital origin are age at onset, presence of constipation and other symptoms from birth as illustrated in all the cases here reported as well as by a study of the pathology. Furthermore, cases have been reported in which fully developed megacolon occurred in prematurely born children. 
Barrington-Ward points out that developmental errors are most common at the junction of the proctodeum and hindgut and that the site of next greatest frequency is at the junction of the foregut and midgut. $\mathrm{He}$ considers that in megacolon the developmental error occurs at the former site, and he draws attention to pyloric stenosis as a possible example of developmental error in the later position. It is well known that the increase in the length of the intestine during the last month of intra-uterine life is usually about 25 per cent., but that there is great individual variation in this respect. At birth the infant's intestine is at least one-tenth longer in proportion to the body length than is the adult's. Much of this increase goes to make the sigmoid of excessive length in the infant. In the normal baby, growth rapidly balances this relationship, but in cases of giant colon such balancing is impossible, at least in well-developed cases. From such facts Finney infers that the colon is still under morphogenetic evolution, that the inconstancy of its length and minor details are but variations in the progressive evolution toward the final arrangement, and that the anomalies are but reversion types. According to him, in megacolon all the segments of the intestine may be involved, but more often the condition concerns that segment whose evolution is most primitive. He lays great stress on the thickness of the mesocolon in one of his cases. This he states to have been the result of enlarged lymph-glands together with enormously dilated lymph and blood vessels, which corresponded exactly with the gigantic section of the bowel. This appearance suggests to him a state of hypernutrition and he calls attention to the fact that this same increase in vessels is a feature of that gigantism which occurs in the limbs; he suggests that the conditions in the bowel may be parallel. A reference to the photomicrographs will show that in our cases very large much-dilated vessels occurred not only in megacolon, but in pyloric stenosis and in giant small intestine as well. But none of our material showed the lymph-node hyperplasia that Finney found in his case.

Briefly stated, Barrington-Ward's theory, which seems the most explanatory, is that there is a congenital abnormality in the lower end of the hindgut which takes the form of a muscular hyperplasia. It usually involves the rectum and spreads a varying distance up the pelvic and iliac colon. It may be that the whole of the hindgut is affected, but in some cases there is a definite localization of the change and the hyperplasia affects only a segment of the intestine. The hyperplasia of the muscles gives rise both to thickening of the walls and to increase of the bowel lumen. The physiologic functions of the intestine are in abeyance, and blocking is produced in the bowel behind, for a varying distance, which depends on the extent and duration of the 
dilatation and hypertrophy. In time this fecal stasis produces necrotic changes in the mucosa, an ulcerative colitis follows which is the cause of death in most instances. It seems reasonable to us that BarringtonWard and Finney each contribute facts that, taken together, explain the underlying causes of the pathology in these conditions.

\section{CASES OF MEGACOLON}

The following cases of congenital enlargement of the intestine are reported because they serve, some to throw light on the disputed points, and others to illustrate the danger of temporizing management, while the last case shows the efficacy of operative interference. The case of microcolon is included not only because of its rarity, but because it illustrates a condition opposite to megacolon and at the same time furnishes us with an abnormality in the small intestine which in every way parallels the pathological changes found in the large intestine in cases of giant colon. We present for comparison the photomicrographs of the giant colon, of the hypertrophied small intestine and of a case of hypertrophic pyloric stenosis. A comparative study of this picture will show how nearly identical the muscle and blood-vessel changes are in the three conditions. Our cases of megacolon are as follows:

Case 1.-Baby S., referred by Dr. A. B. Spalding. Seen on the fourth day of life. The mother and father were healthy people. The birth normal, at full term. The child was brought in because of increasing abdominal distention and because the bowels had not moved since just after birth, when a small amount of mucus and meconium-like material was passed. No one had noticed the passage of any flatus.

On admission, the child weighed 8 pounds. It appeared somewhat driedout, the skin hanging loose and lusterless. The child was well formed with no deformities except that the abdomen was exceedingly prominent. This enlargement was especially marked when contrasted with the dried-out limbs. and was taken to be due to gas retention. It was noted that there was an area of visible intestinal peristalsis which passed across the abdomen from the left iliac fossa to the border of the ribs on the right side. This area was horseshoe-shaped and seemed to be due to movenents in two separate segments of the intestine. The upper segment showed movement from left to right, while the movements in the lower segment appeared to pass from right to left. On examination the heart and lungs were normal; no tumors were determined in the abdomen. The abdomen was distended but not hard and was easily pal. pated. The anus was patent; the little finger entered an apparently normal rectum but was arrested just above the brim of the pelvis by what felt like a narrow band.

The child retained milk from one-half to one hour and then rejected it all. Attempts to give enemas were unsuccessful; the water returned uncolored. A bismuth meal was given and the child radiographed by Dr. Boardman. In the picture the stomach appeared normal, but the plates taken one and two hours after the meal showed no retention of bismuth in either stomach or intestine; all had been vomited. The child was kept alive by hyperdermoclyses of 4.5 per cent. glucose in normal salt solution.

Based on the finding in the rectum of a definite narrowing and of an apparcnt obstruction to the exit of the bismuth meal from the stomach together 
with risible peristalsis, a diagnosis wats marte of multiple stenosis of the intestinal tract with narrowing at the duodenum in the small intestine and at the pelvic colon.

A surgeon was asked to see the chili and to attempt multiple anastomosis. When the ahdomen was opened the true state of affairs was revealed. A giant colon bulged into the wound and was returnable with great difficulty. The area of peristalsis proved to have been produced by a long redundant loop of the sigmoid which stretehed across the ahdomen. The outgoing loop crossed the transwerse colon as high as the plorus; it then wound around the lower
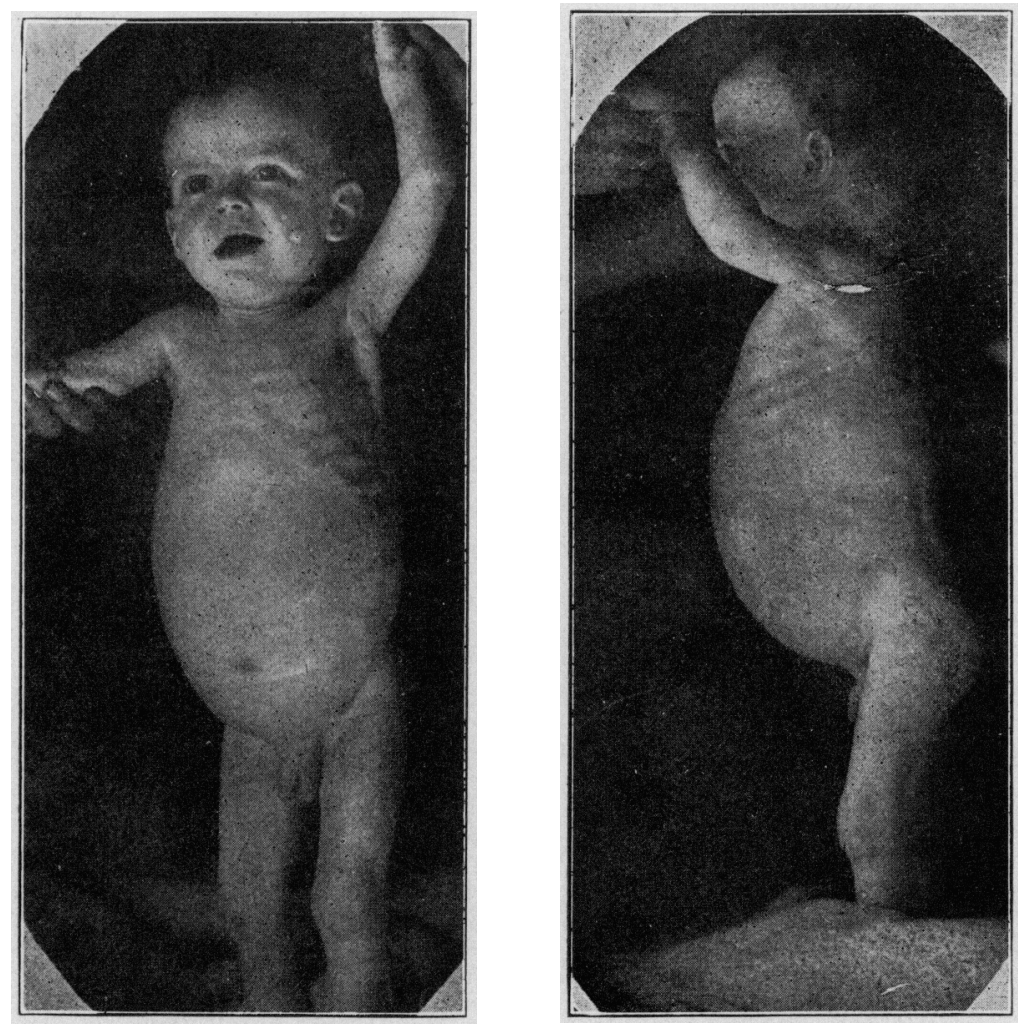

Figs. 1 and 2.-Patient 3, three monthe hefore death from pertussis.

border of the liver, and the incoming loop, starting aloove the hepatic flexure, ran thence to the left iliac fossa, where it terminated in a distinctly narrow 1,ut slightly hypertrophied area about $1 \mathrm{t} / 2$ inches in length. The child succumbed to the operation. Necropsy contirmed the lindings revealed at the operation.

Cnfortunately, the removed intestine was lost, hence it is impossible to report on the microscopic conditions present in this specimen.

This case is of interest in that it chows true giant colon to be a condition that may present itself at birth with fully developed dilatation and hypertrophy - this in spite of the view of those atthors who 
holl the dilatation to be variable and secondary, and others who have a like opinion about the hypertrophy. It is further of interest because, had the diagnosis been made and the intestine promptly opened for drainage, the child might have survived, and a later attempt could have been made to remove the large intestine, as has been done by Arbuthnot Lane. The way in which young children respond to gastro-enterostomy for pyloric stenosis is warrant for the faith that this child might have survived operative interference.

Case 2.-A. IF., 18 months of age. Seen in the conrse of private practice with the history of having had obstinate constipation and "big stomach" since the fourth month. He was the first and only child, born at full term, after protracted labor. The child had been difficult to feed in that he had not

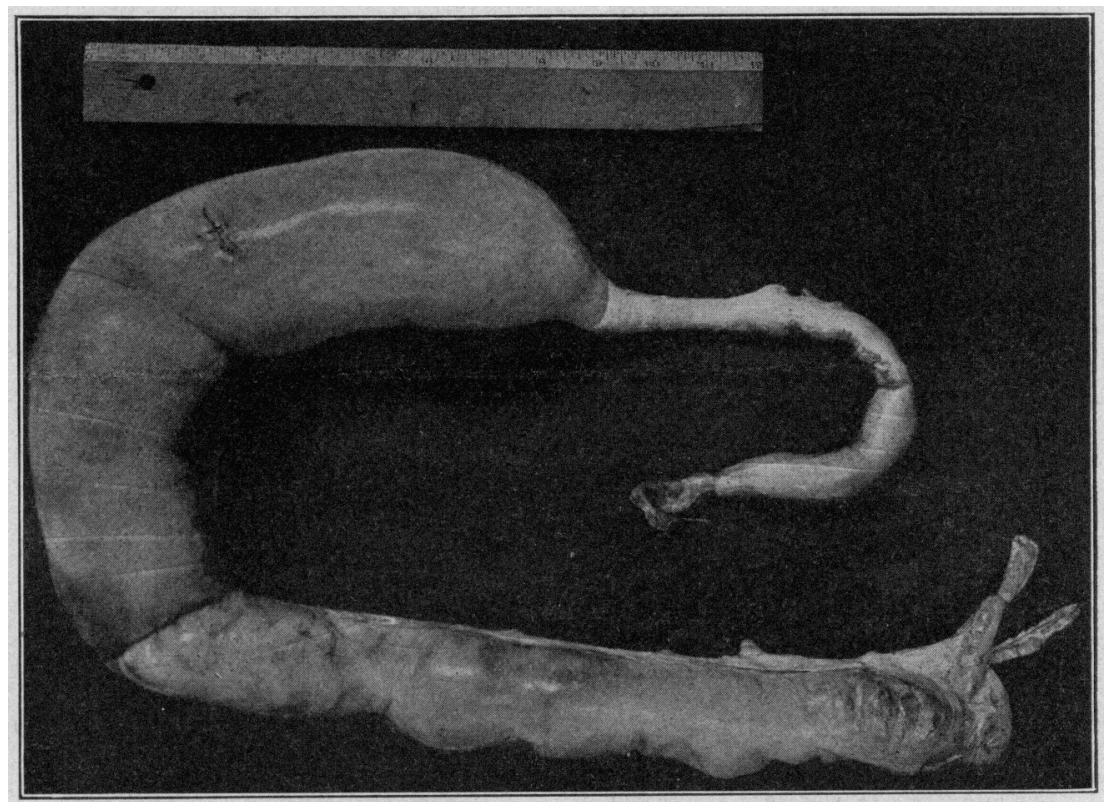

Fig. 3.-Colon from Case 3.

thriven on varying formulas and was astoundingly constipated. On one occasion, during the sixth month, he had passed several days (the mother said ten), whont cvacuation, and on many occasions there were periods of from two to five days without bowel movencuts. At such times the abdomen became tremendonsly enlarged beyond its ordinary huge size and the child would suffer attacks with rapid, shallow pulse, milarrassed breathing, and threatened collapse. The mother had learned that hy the airl of a soft rubber catheter she conld manipulate the contents of the bowel and so greatly relicre the child of the accunnlated gas, and with this manewer she carried the patient through many stich crises. She harl also discovered that the blocking of the bowel by feces was relicved and the gas most readily passed when the child"s hips were held high above the shoulders. It is possible that in this position the retained mass was freed from the narrowed sigmoinl by the aid of gravity.

On examination there was revealed an cmaciated child with a huge abromen, measuring twenty-four inches at the umbilicus, and looking. as one colleague 
remarked, like a halloon tied to a little skeleton. The skin was dry and hung in folds everywhere except from the abdomen. The distention of the abdomen was exaggerated at the flanks, where the bulging was cxtrotorlinary. The diaphragm was high, and the costal region very wide. Fo visible peristalsis was noted. Masse's, hard but cloughy, were folt in the abclomen, one the size of a large orange on the right above the navel, another in the left iliac region about the same sizc, and smaller ones lay in what seemed to be the line of the transerse colon. These were moved about and felt with difficulty because of the extreme dictention. Rectal examination revealerl a wide, lower rectum. the little finger was stopped at about the polvic rin by what seemed a detinite

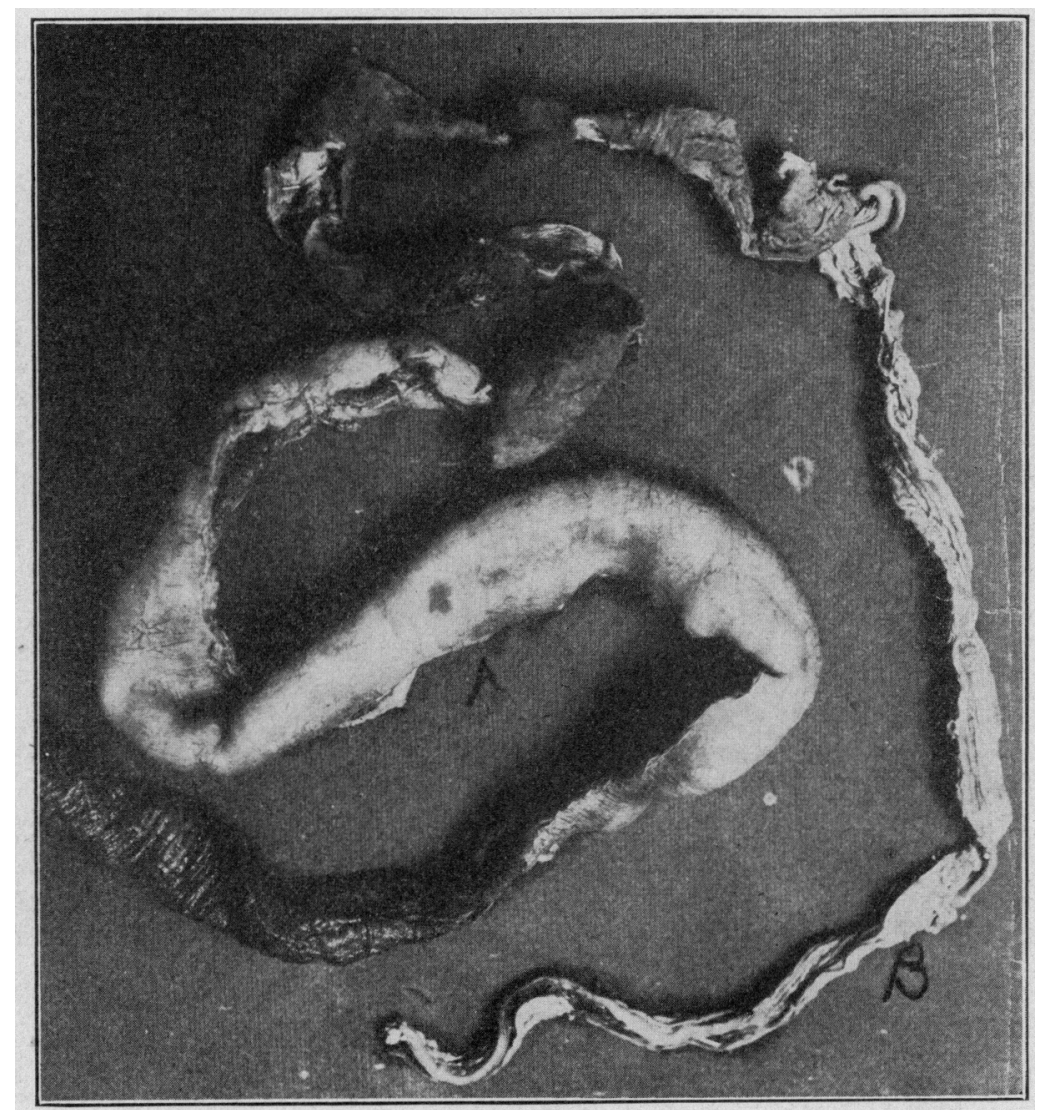

Fig̣. 4.-Intestines from case of microcolon. A, gigantism of small intestinc; $B$, microcolon.

narrowing. Pimanual palpitation defined a tumor in the left iliac region, but presure could not force it lownward through the constriction.

The child now cutered the Lane Hospital and it was decided to wait twentyfour hours and then to create an artificial anus. The pationt seemed in excellent condition. Glycerin and water enemas were ordered and an attempt was made to produce cvacuation. Several such enemas were given without result in the course of twelve hours and cach tine all of the fluid was returned unstained, according to the nurse's report. Apparently. lowwerer, a mass of 
feces in the transverse colon was dislodged and dropped down into the narrow part of the intestine, where it produced an acute obstruction. At about $10 \mathrm{p} . \mathrm{m}$. on the day of the child's entrance into the hospital, one of us was summoned hurriedly to find the child in extremis with the ptilse running shallow and uncountable, the face gray, the lips blue, and the patient barely conscious. There was the most tremendous distention conceivable. In the absence of Dr. Weeks from San Francisco, a hurried summons was sent to another surgeon, but before he could reach the operating-room the child was dead. Obviously, the thing which should have been done was to incise, draw out the intestine and open. In the absence of surgical experience, and in the hope of finding skilled aid at once, one unskilled in surgery hesitated to assume this responsibility, but, undoubtedly, if such an emergency were faced again, the opening would be made without hesitation.

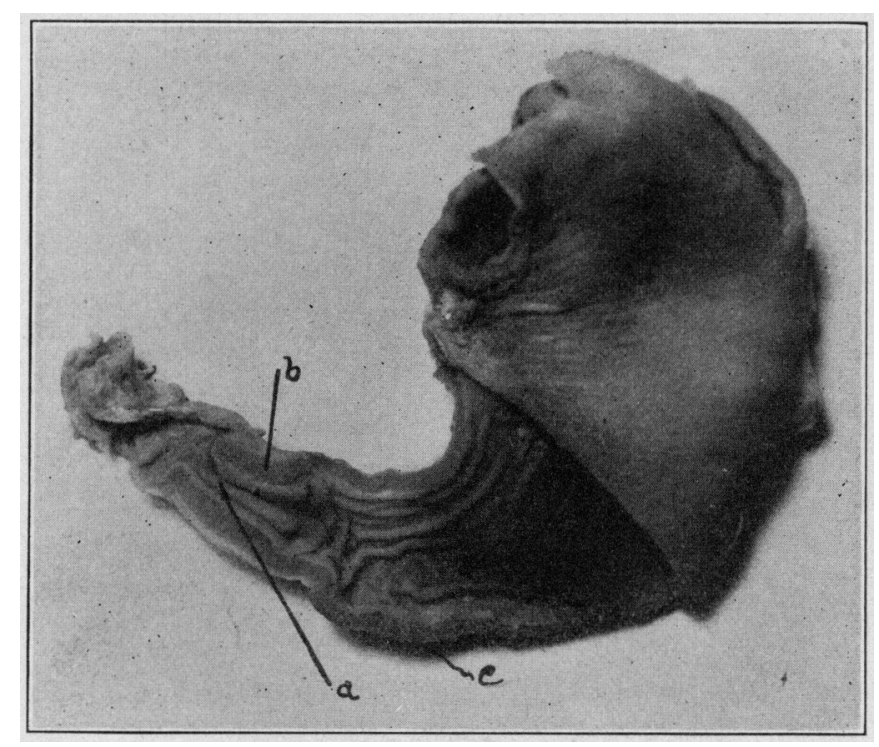

Fig. 5.-Hypertrophic pyloric stenosis, from photograph, full size; $a$, narrowing of lumen; $b$, ring muscle; $c$, hypertrophy of circular muscular coat of stomach. The narrow lumen of the pylorus is seen to be almost completely occluded at $a$. The wall of the pylorus is seen to be greatly thickened at $b$, and is continuous with the hypertrophied musculature of pyloric antrum.

Necropsy was denied us in this instance, so our only help from it lies in the illustration of the potential dangers of the condition and in the emphasis it puts on the view that the only safe procedure in these cases is to make a preliminary colotomy with artificial anus at the earliest possible moment, so that the intestine may be emptied of its retained material. After such procedure the poisoning ceases, the child's nutrition improves, and, with a wait of from two to four months, the physical conditions are so mended that there is warrant for a resection of the entire large intestine with the hope of permanent cure. 
CASE 3.-E. S., male, 19 months of age, weight 15 pounds and 4 ounces, seen in consultation with Dr. Dudley Smith (Figs. 1 and 2). Full term, normal birth, birth weight $73 / 4$ pounds. The abdomen was so distended at birth that the parents thought the child's hips were deformed because of the great contrast. Constipation at that time was very marked, and there was no evacuation until the fourth day of life, when scybalous masses of hardened meconium were passed, but in small quantity. In the second month a period of nine days elapsed without any movement of the bowel; the abdomen was greatly distended, and the child was much distressed. Evacuation was finally accomplished by enemas and castor oil, but these agencies produced only small marble-like scybala. At the time of this attack no signs of collapse supervened with the distention. The physician then in charge considered the condition to be due to unsuitable food, and the child was subjected to numerous changes in diet without effect. He gained slowly in weight and strength, but such attacks as just described recurred almost every month. On one occasion in the fourth month there was marked diarrhea which lasted for a week and was accompanied

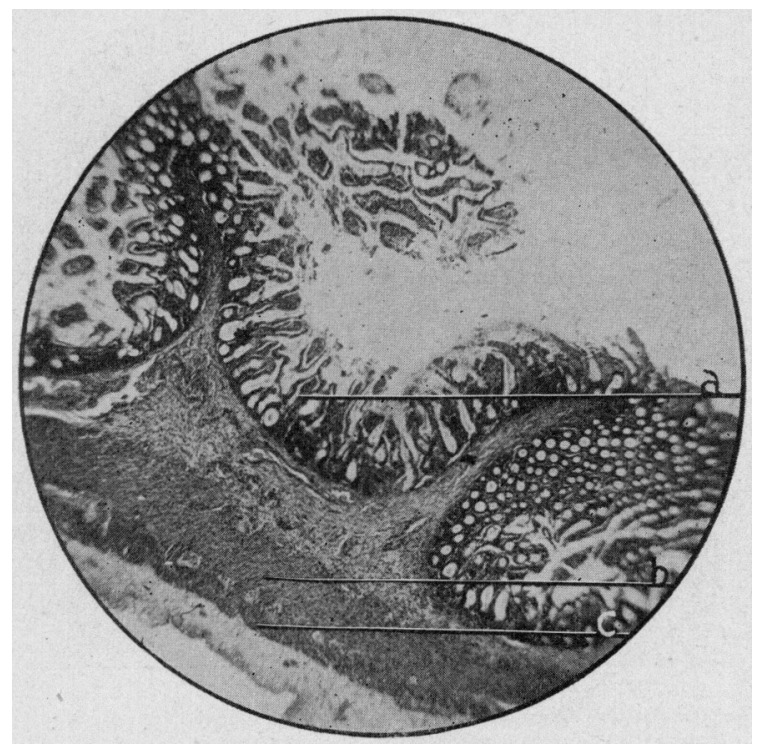

Fig. 6.-High-power photomicrograph of the hypertrophied ileum. The normal mucosa is seen at $a$, under which lies the somewhat thickened submucosa. The muscular layers ( $b$ and $c$ ) are hypertrophied, especially the internal coat. The serosa, lying external to the muscular layers $(c)$ is edematous.

by depression and great distress, which was relieved by castor oil and turpentine enemas. At six months, after obstruction of four days' duration, a characteristic attack supervened with pain, distention and collapse; at the eighth month another similar attack came on.

Dr. Dudley Smith now saw the child for the first time. He was able to effect evacuation of the bowels and to keep the child in fair condition by large doses of magnesia and the passage of a soft rubber catheter, which effectively relieved the distention. The child's nutrition began shortly to improve; his weight curve commenced to ascend and he reached $19 \mathrm{I} / 4$ pounds in the seventeenth month, in spite of the fact that severe obstructive attacks occurred during the fourteenth and sixteenth months. During the eighteenth month the boy's weight fell during such an attack to $153 / 4$ pounds, when one of us saw him for the first time and we coincided with Dr. Smith's opinion that oper- 
ative interference held the only promise of relief for the condition. This help was not to be, however, as the child was even then in the first stage of pertussis, to which he later succumbed. The necropsy revealed a typical megacolon (Fig. 3).

The physical examination of this child showed simply a distended abdomen in a rickety subject who exhibited visible peristalsis over the middle and upper section of the abdomen; there were palpable fecal masses, and the rectum on examination revealed a narrowing at the pelvic brim. This case, like the last, shows the method of obstruction that occurs in this pathologic state and in each instance, the pain, collapse, and obstruction were relieved after the passage of a hard, narrow fecal mass which apparently had lodged in the narrowed sigmoid.

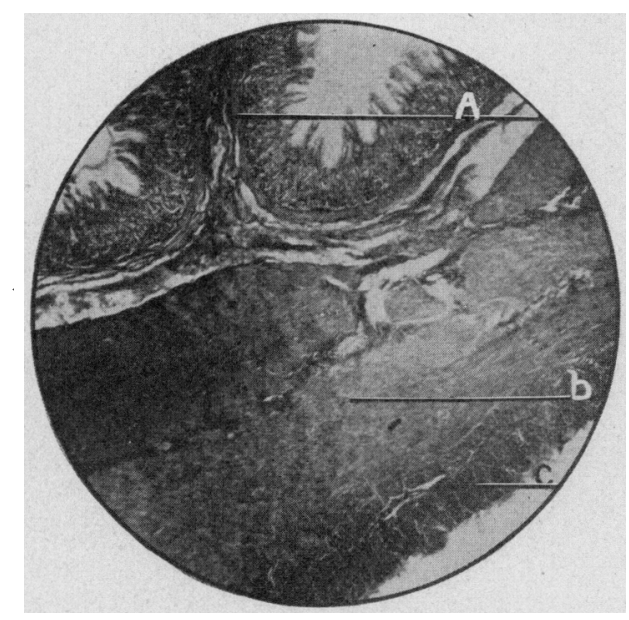

Fig. 7.-Photomicrograph of hypertrophied wall of pyloric antrum. The mucosa $(a)$ is normal and is covered with a layer of mucus. Beneath it lies ths submucosa, which is loosened from the underlying internal muscular coat (b), which shows a marked hypertrophy. Throughout it are seen fibrous connective tissue strands extending down from the submucosa. The external muscular layer $(c)$ is not much thickened.

CASE 4.-E. P., male, 22 months of age, referred by Dr. Hughes of Sonoma. Cal., with the diagnosis of Hirschsprung's disease. This was the second child, full term, normal birth. Parents and other children normal and in good health. Symptoms were not noticed by the parents until the fourth month of life. From that time the history was like that of the other cases reported, malnutrition and difficult digestion with attacks of obstruction and collapse. On examination we were confronted with a yellowish gray-skinned anemic child, weighing 22 pounds. There was an enormous abdomen, which measured thirty inches at the level of the umbilicus. We saw no visible peristalsis on this occasion, and there was no such tenseness of the abdomen as had been met in the other cases. Palpation of the abdomen revealed several tumors; the largest, the size of a fetal head, lay in the right iliac fossa, extending upward and toward the right where the bulging was marked. Other similar masses lay above the umbilicus and to the left of it. The largest mass was 
smooth, easily movable and was taken to consist of feces lying in the caput coli. Rectal examination revealed no narrowing; on the contrary, the rectum and colon were very large and roomy. Previously, a diagnosis of tuberculous mesenteric adenitis had been made. Bimanual examination with the finger in the rectum revealcd a smooth, velvety peritoneum and contradicted this assumption.

Dr. Weeks now saw the child for the first time and advised operation, proposing a two-stage procedure. At the first operation the abdomen was opened on the left side and an artificial anus was created. The method of Levison of San Francisco was utilized and the retained fecal masses were then evacuated and we found that the one which had been discovered on the right side was lying, not in the caput coli, but in the redundant loop of the huge sigmoid. This giant sigmoid crossed over the abdomen and filled most of it; it overlay the apparently normal caput coli and thus gave rise to the erroneous impression. The cperator drew the intestine down as far as possible into the left iliac fossa, where it was fastened, opened, and its contents evacuated. The child did well, the toxic symptoms manifested in the dry, yellow skin, anemia.

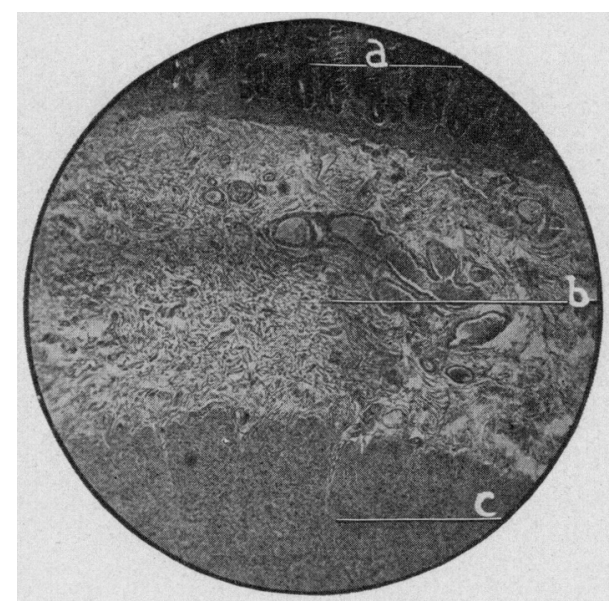

Fig. 8.-High power photomicrograph of megacolon. The mucosa at $(a)$ is normal. The submucosa $(b)$ is markedly thickened and fibrous and contains many large dilated vessels filled with blood. The muscle layer (c) is also thicker than normal.

and poor nutrition gave way, and at the end of six weeks the child had gained 12 pounds in weight. For four months evacuation was through the artificial opening, then as the child had made such great improvement, it was decided to resect the intestine. This was done at the Children's Hospital in San Francisco. Under ether the redundant sigmoid and the intestine above it up to what appeared to be the normal first part of the transverse colon were resected. After removal this redundant loop was 15 inches in length and in diameter about 3 inches, and its walls about $5 / 16$ of an inch in thickness. The freed upper end was stitched into the severed end of the rectum; this was accomplished with great difficulty because the rectal tissues were thick from hypertrophy and the tube at that point was nearly three times as wide as was the implanted end of the large intestine.

The report of the microscopic section (Figs. 10 and 11) by Jean Oliver is as follows: "The sections show a normal mucous membrane with well-marked lymphoid follicles which are somewhat hyperplastic. All the muscular elements of the intestine are markedly hypertrophied, including the muscularis mucosae. 
The submucosa is rather fibrous and the submucosa and serosa normal. Many large dilated blood-vessels filled with blood are found."

The anastomosis was successful except that there was a small area of imperfect union which gave rise to a slight fecal fistula. This opening, however, closed spontaneously after about two weeks and the patient went on to apparent recovery with occasional attacks of retention of feces and moderate

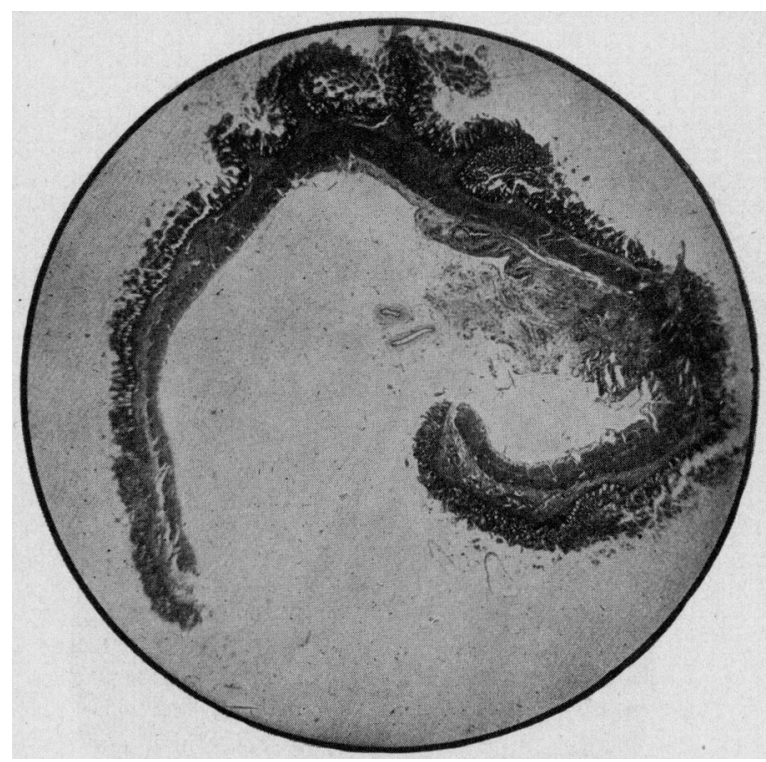

Fig. 9.

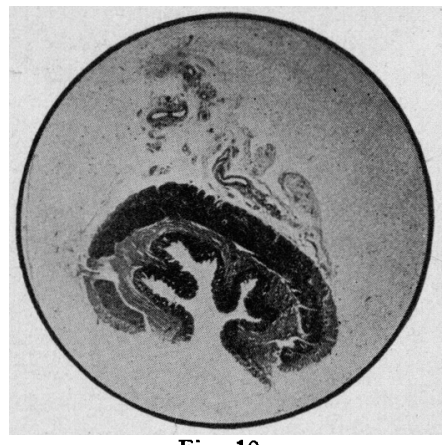

Fig. 10.

Figs. 9 and 10.-Low-power photomicrographs of the microcolon (Fig. 10) and the dilated and hypertrophied ileum (Fig. 9) showing the relative size of the gut of the different levels and the thickness of its walls. The marked dilatation of the ileum (Fig. 9) masks to a certain degree the hypertrophy of the musctilar coats so that they do not appear much thicker than those of the microcolon (Fig. 10).

abdominal distention, which were apparently due to the fact that the huge rectum was deficient in tone and expulsive power. For some months the improvement was marked and progressive, but at no time has the patient done so well as during the period while he carried a freely opened artificial anus. 
The boweis have to be evacuated by the aid of small low enemas of glycerin or soap and water. This dislodges what appears to be a normal fecal mass from the rectum and each enema is followed by a normal-appearing evacuation.

Six months after the operation the mother stated by letter that some distention was recurring. This is undoubtedly due to the fact that the portion of transverse colon which was left suffered from the same pathologic defect as the portion amputated, although probably to a less degree, and this region is now giving way under the stress of pressure. It seems probable that the remaining colon will yet have to be removed.

\section{CASE OF MICROCOLON WITH HYPERTROPHIED SMALL INTESTINE}

During the period of our interest in megacolon Baby R., male, 5 days of age, was brought into the Lane Hospital in extremis, deeply jaundiced and cyanotic, incessantly vomiting, ejecting meconium-like material. The child had had no evacuation since hirth, had taken but little food and had passed urine a few drops at a time only. The vomiting had persisted for forty-eight hours before entrance. The abdomen was distended and tense; it was possible to diag-

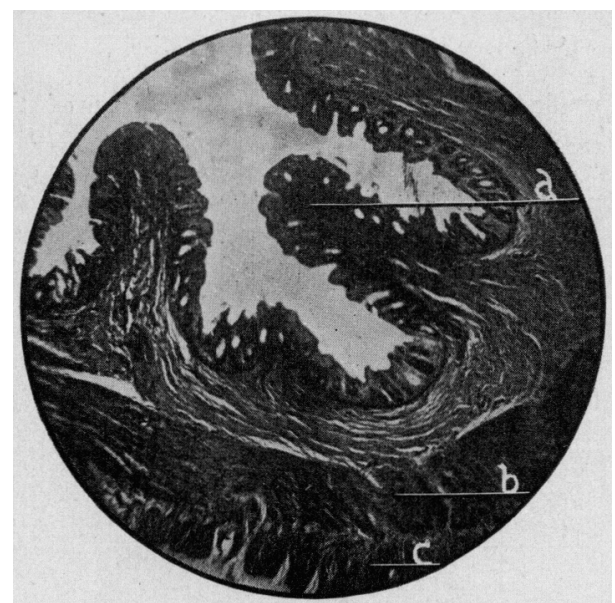

Fig. 11.-High-power photomicrograph of microcolon. The mucosa $(a)$ is normal, and the underlying submucosa is somewhat fibrous. The muscular layers $(b$ and $c$ ) are relatively thicker than normal, but the decrease in the size of the intestine is evident in all its layers, no single layer being decreased at the expense of the others.

nose the presence of free fluid in the peritoneal cavity. The anal orifice was normal and the little finger moved freely into the rectum and was stopped just above the pelvic brim by a marked narrowing. A diagnosis of intestinal obstruction due to stenosis at the sigmoid was made, but the child was past aid and died an hour after admission. The necropsy was performed by Dr. William Ophüls, whose notes are as follows:

"Strongly built, male baby, $54 \mathrm{~cm}$. in length. Cyanosis. Anus well formed, patulous. No obstruction in any of the natural orifices. Skin shows yellowish hue all over.

"Some gas in peritoneum and about 350 c.c. of distinctly fecal fluid. Peritoneum is inflamed and covered with soft greenish shreds of fibrin. Omentum is small, hyperemic, contains no fat. Transverse and descending colon very thin and contracted. Sigmoid flexure is pulled up out of pelvis to a certain extent and fastened by vascular adhesions to the mesentery of one of the loops of small intestine. These adhesions are below the point of greatest distention of the bowel. About $10 \mathrm{~cm}$. above the ileocecal valve is a small perforation. 
"Upper part of small bowel distended with gas, lower part very much distended; diameter of intestine below is $3.5 \mathrm{~cm}$. in places. Further down distention decreases, increases again, and at the ileocecal valve the intestine has normal diameter.

"Above-described adhesion is below the point of greatest distention of bowel; about $10 \mathrm{~cm}$. above the ileocecal valve is an oval perforation, about $5 \mathrm{~mm}$. in diameter, situated close to the mesenteric attachment, through which fecal matter escapes.

"Upper part of the small bowel is filled with soft greenish brown fecal material. In lowest part of small bowel is thick, pasty material. The ileocecal valve is open. The large intestine is very much contracted. It is almost empty but in some places there is a small amount of dried-up mucus. The rectum is of normal caliber, its mucous membrane hyperemic, with some blood in rectum. Appendix is normal. In region of perforation mucous membrane shows marked hyperemia and extensive hemorrhages, and there is some evidence of necrosis of the mucous membrane. Very much distended part of lower small intestine shows well-marked hypertrophy of muscular wall, being almost $4 \mathrm{~mm}$. thick in places. Mucous membrane is distended, part much inflamed, superficial erosions in places. Necropsy findings revealed no other abnormalities."

Sections were made from the hypertrophied areas of the small intestine by Jean Oliver, whose report is here given.

"Section of the small intestine through its enlarged part shows marked hypertrophy of the muscular layers. This is especially prominent when one considers that there is also great dilatation, which tends to obscure the hypertrophy of the walls. The mucous membrane is extremely hyperemic and there are many large hemorrhages, especially in the submucosa. A considerable layer of mucus covers the epithelium. The serosa is also hyperemic and there is beginning cellular infiltration and deposit of fbrin on its surface. The bloodvessels are large, dilated and filled with blood.

"Sections of the microcolon show the same hyperemia and hemorrhages in the mucous membranes but to a less degree. The submucosa is quite fibrous and also shows considerable diffuse hemorrhage. The muscular layers appear thickened, probably owing to the contraction of the intestine. The subserosa shows marked cellular infiltration and there is a heavy deposit of fibrin in the serosa."

The case of microcolon is included in these reports, not only because of its striking contrast to the megacolon cases, but also because there was also present a condition of hypertrophy of the small intestine (Fig. 4) in which the changes in the muscle and fibrous tissues parallel in every pathologic way the changes of the analogous tissues in the large intestine found in the cases of Hirschsprung's disease. It always seemed to us that the innate pathology underlying megacolon is akin to, if not identical with, the changes in tissue found in hypertrophic pyloric stenosis, especially in that form wherein there is general hypertrophy of the circular muscle of the stomach wall as well as of the ring muscle at the pylorus (Fig. 5). This case of microcolon with gigantism of the small intestine provides a true example of the same marked growth of muscle and hypertrophy of fibrous tissue with gigantism of vessels occurring in the small intestine.

Microphotographs of the sections of the three conditions have been made in the standard Pathological Laboratory by Jean Oliver (Figs. 6, 
$7,8,9,10$ and 11). A study of them will reveal how like the muscle affection and the marked hypertrophy in the mucosa connective tissue and vessels is in the three conditions. The photograph of the stomach from a patient with pyloric stenosis also is evidence for this point of view.

The consideration of our cases of megacolon gives some evidence on disputed points. Certain writers have denied the congenital origin of this condition. Our Cases 1 and 3 exhibited symptoms at birth and in Case 1 a typically developed giant colon was revealed by operation and necropsy. Cases have been reported in which giant colon was discovered in prematurely born children.

It has been questioned whether the sigmoid was narrow in all cases, and it has been asked if the true origin of the dilatation and hypertrophy was not an obstruction that blocked the passage with feces and allowed the work of the intestine and the stress on it to be followed by increased production of muscle and ballooning from the retained gas. Case 4 of this series, in which the gigantism included every portion of the intestine to the anus, shows that the sigmoid narrowing is not present in every case. Case 2 is instructive in demonstrating the potential and emergent dangers to life of the condition, and with Case 4 causes us to resolve that in the future even the youngest child encountered with this trouble shall have the benefit of immediate colotomy and an artificial anus. The well-being of Patient 4 during the time he carried an artificial anus was so striking and the operation is so easy that these facts, taken with the well-known tolerance of infants to abdominal interference, make such an operative interference most reasonable, especially when we consider the unhappy course of Case 2 which demonstrated so completely the extreme danger of temporizing and of trying to evacuate the bowel through the rectum in those cases of megacolon with a narrow sigmoid.

As one considers the condition of gigantism in the intestine, the question presents itself: Are there mild cases of the disease? One who deals much with infants cannot but feel that such cases occur, and are not very unusual. There are obstinately constipated infants, who, while not rachitic, have abdomens that bulge inordinately and in whom the bulging for the most part follows the line of the colon and fills out the flanks to a marked degree. Such children are apt to be uncomfortable, to take food badly and to fail in weight, but they are not distressed by flatus. When their bowels are kept free all the symptoms disappear except the ballooning of the large intestine, and growth seems to adapt the children in time even to this visceral enlargement, for the individuals in this group seemed to do well once they are past infancy. The final proof of this contention must rest on 
necropsy of such a child who has met death from another cause, as the condition itself is never fatal, nor indeed is it severe. Hertz has called attention to a class of infants in whom roentgenograms show a lengthened sigmoid, and it may very well be that such infants correspond in the group of megacolon to the cases of mild hypertrophy with spasm that we encounter in the pyloric stenosis group.

The proper feeding of infants with congenital enlargement of the colon is of importance and presents difficulties. High fat formulas are not adapted to this condition, as soap stools give more trouble in all classes of megacolon than do other types of stools. Giant colon cases seem to respond to high percentages of dextrin, yet care must be exercised, as fermentation and gas formation have to be discouraged. Enemas are on the whole the best means of producing bowel evacuations. Castor oil is to be shunned, as is magnesia. In older children milk should not be given.

The clinical picture of congenital enlargement of intestine and the colon is so characteristic that it seems hardly needful to discuss the differential diagnosis. The one case in our series which led to confusion was that of the new-born infant. The differentiation between multiple stenosis and megacolon in the new-born is certainly difficult, but the character of the peristalsis and the mildness of the digestive symptoms should have been a guide to a proper diagnosis. This case was rendered more difficult by the vomiting, which was extreme, and which probably is a most unusual accompaniment of congenital enlargement of the intestine. The only other condition which might possibly lead to a mistake in diagnosis is tuberculous peritonitis, and this mistake will never be made if the child is examined under an anesthetic by the bimanual method, a finger of one hand in the rectum and the other hand on the abdominal wall. The characteristic smooth feel of the healthy peritoneum instantly excludes the presence of tuberculous inflammation. The gristly fibrous bands and the rolled-up lower edge of the omentum in tuberculous peritonitis give to the examining finger a sensation that is absolutely pathognomonic. (In passing, it may be said that there is no maneuver in diagnostic examination of children of more value than this bimanual rectal examination if it be made while the child is under the influence of an anesthetic.) It is unlikely that any of the abdominal tumors will be confused and hardly possible that the dilatation due to chronic intestinal indigestion in rickety children will mislead one.

It is probable that megacolon is a more common deformity than is generally thought. The symptoms are present from birth. They are: an enlarged abdomen, visible intestinal peristalsis, obstinate constipation, later, anemia and toxemia from fecal retention, accompanied 
sometimes by recurring attacks of intestina! obstruction. Pathologic hypertrophy with dilatation may include the entire intestine, or the dilatation may cease at the sigmoid, which will be hypertrophied, but with a narrow lumen. That isolated segments of the large intestine may be affected while most of the intestine remains intact and healthy is to be doubted. Fatal collapse can occur from fecal obstruction, and such obstruction may be brought on by injudicious attempts to empty the bowel. The one measure that promises relief is operative procedure, which may be utilized in either two or three stages: colotomy, with artificial anus (this cannot be done too early in the child's life or in the course of the case), later excision of the entire large intestine with anastomosis from the ileocecal region into the rectum; or anastomosis may be done as a second step and resection of the entire colon be'made a third operation.

240 Stockton Street-350 Post Street. 